\title{
The Combination of Diffusion Model and Learning Effect Model to Evaluate the Policy Impact of Cancer Control Act
}

\author{
Y.T. Chen \\ National Taipei University of Nursing and Health Sciences, Taipei, R.O.C. \\ F.C. Yang \\ Delin Institute of Technology, Taipei, R.O.C.
}

\begin{abstract}
Considering the social high incidence and mortality rate of cervix cancer, the government provides a free medical service of Pap smear test based on the Cancer Control Act regulated in 2003. This paper attempts to understand the impact of policy implementation for Pap smear test and employs the diffusion model, learning model and integrating model respectively to examine the diffusion of Pap smear test promotion. The results show that all the three models are statistically fitted, i.e. diffusion effects, learning effects, and interaction effects exists and may explain the trend of Pap smear test. The policy of the Cancer Control Act is also found to significantly affect the adoption of Pap smear test.
\end{abstract}

KEYWORD: Diffusion Model; Learning Model; Integrating Model; Cancer Control Act

\section{INTRODUCTION}

With the rapid economic development of society, the lifestyles change fast and also bring many serious public health problems in Taiwan. Since 1982, malignant neoplasm has become the first leading cause of mortality in Taiwan. In order to retard the high mortality causing from cancer, Department of Health implements serious countermeasures and regulates the "Cancer Control Act" as a guideline to enhance the rate of cancer screening. Chen et al. (2007) propose that cancer screening is a powerful tool to detect cancer and may provide a highly effective medical treatment for curing cancer in the early stage. The National Health Insurance (NHI) program offers the free service of Pap smear test for every female aged 30 and above, and the government also offers some service that may minimize the financial barrier for every female in Taiwan. The free Pap smear test is treated as a new service provided by government in this paper. Government dominates Pap smear test promotion by implementing policies while individual stakeholders are regarded as adopters of such new service. The government has to keep continuous efforts to enhance the performance of organization by expanding the employee capacity through the reform of management system and the improved organizational learning.

In this paper, the performance of Pap smear test promotion may attribute to diffusion effect and organizational learning effect. Montalvo and Kemp (2008) propose that diffusion can be treated as an adoption process of new technology by population in social networks overtime and the process implies that the potential may adopt the new innovative product by learning the existence information through the direct relationship between a potential adopter and a user. The performance of organization can be improved by experience and the activity of organizational learning that includes the acquisition of explicit and tacit knowledge, knowledge sharing, and knowledge use (Chen and Chang, 2010).

The communication between users and potential adopters is the main factor as technology diffusion. We treated females as adopter population for Pap smear test when learning effect takes place within the organizational structure of Department of Health. We also discuss the policy and other factors that may affect the diffusion process by incorporating these factors into model. In addition, we discuss the interaction effect between the diffusion effect and organizational learning effect and analyze the role of interaction effect that affect the promotion of Pap smear test. We propose that interaction effect may influence the promotion of Pap smear test by integrating system including National Department of Health and females, and bring about the success management in National Department of Health.

\section{METHODS}

\subsection{Diffusion model}

The diffusion process implies a communication process that spreads the information between the 
users and potential adopters, and thus the process may change the behaviors and perception of potential adopters by imitation and learning (Chen and Chang, 2010). The Bass model describes the diffusion pattern for the analysis of innovation diffusion (Bayus, 1993), expressed below:

$$
\frac{d n(t)}{d t}=p[M-n(t)]+q \frac{n(t)}{M}[M-n(t)]
$$

where $n(t)$ is the accumulated adopters, $t$ represents time, $p$ and $q$ are coefficients of external and internal influence respectively, and $M$ is the ceiling of adopters. In Bass model, potential adopters may be influenced by two types of communication channels: mass media and interpersonal factors. The information is spread through mass media to attract the number of nonadopters in the system. In this paper, the factor of mass media is assumed to be neglible and thus the term of $p[M-n(t)]$ on the right-hand side of Eq. (1) is removed. Hence, a logistic model describing the process of innovation adoption is employed and expressed in Eq. (2) (Chen and Chang, 2010).

$$
\frac{d x}{d t}=\alpha x\left(1-\frac{x}{F}\right)
$$

where $x$ is the number of Pap smear test, $\alpha$ is the natural growth rate of diffusion process with $\alpha>0$, $t$ treats as the time, and $F$ is the potential carrying capacity, defined by the amount of female in a society. We assume that $F$ is given and fixed, treated as a parameter. The government looks forward to promote the service for all females aged over 30 in Taiwan. Therefore, all female aged over 30 can be treated as the number of the adopters and potential adopters for the service of Pap smear test. Converting Eq. (2) yields a difference equation, expressed below:

$$
\left(x_{t}-x_{t-1}\right)=\alpha x_{t-1}\left(1-\frac{x_{t-1}}{F}\right)
$$

Chen and Chang (2010) adopt a time-varying parameter $\alpha$ that may bring substantially better fits and lower forecasting errors. Since policy implementation may bring a positive impact on the process of diffusion, it is employed as a factor to affect parameter $\alpha$. In order to increase the amount of Pap smear test, our government implements the policy of Cancer Control Act by integrating and utilizing health and medical care resources for the effective promotion of cancer prevention in 2003. Thus, parameter $\alpha$ is defined as time varying and influenced by the policy implementation of Cancer Control Act, i.e.

$$
\alpha=\alpha_{0}+\alpha_{1} I_{1}(t)
$$

where $I(t)$ represent the policy of Cancer Control Act and $I_{1}(t)=1$ for the year after year 2003, otherwise $I_{1}(t)=0$.

Substituting Eq. (4) into Eq. (3) yields Eq. (5), listed below:

$$
\begin{aligned}
& x_{t}=\beta_{1} x_{t-1}+\beta_{2} I_{1}(t) x_{t-1}+\beta_{3} x_{t-1}^{2} \\
& +\beta_{4} I_{1}(t) x_{t-1}^{2}+\beta_{5} I_{2}(t)+u_{t}
\end{aligned}
$$

where $\beta_{i}$ is the coefficient of variables, $I_{2}(t)$ represents the event of SARS occurred in 2003, because of the temporary influence from SARS, we set $I_{2}(2003,2004)=1$, otherwise $I_{2}(t)=0$, and $u_{t}$ is the error term distributed by a normal distribution function with zero mean and variance of $\varepsilon_{1}^{2}$.

\subsection{Organizational learning model}

In recent years, the research that focuses on the relationship between organizational learning and organizational performance has grown exponentially and the positive effect of organizational learning on effect performance has also been proved (BolívarRamos et al., 2012). Therefore, the organizational learning model can be expressed as follows:

$$
x_{t}=A\left(\sum_{0}^{t-1} x_{s}\right)^{k}
$$

where $k$ represents learning coefficient. We take a logarithm on both sides of Eq. (6) yields:

$$
\ln x_{t}=h_{0}+h_{1} \ln \sum_{0}^{t-1} x_{s}+v_{t}
$$

where $h_{0}$ refers to $\ln A$ and $v_{t}$ represents an error term, distributed over a normal distribution with zero mean and variance of $\varepsilon_{2}^{2}$.

\subsection{Interaction model}

Meyer and Ausbel (1999) propose that some factors of diffusion path may use to promote the diffusion. Kemp (1998) proposes that learning is an endogenous factor that affects the process of diffusion. In practice, learning effect may bring the influence to the performance of International Health Department and affect the perception of female to accept the new service of Pap smear test. According to the model proposed by Chen and Chang (2010), the interactive relationship between the adopters and organization can be described by interaction model resulted from rearranging the Eq. (1).

$$
\ln x_{t}-\ln x_{t-1}=\alpha\left(1-\frac{x_{t-1}}{F}\right)
$$

We integrate Eq. (8) with (7) can obtain the interaction effect model. 


$$
\begin{aligned}
& z_{t}=\gamma_{0}+\gamma_{1} x_{t-1}+\gamma_{2} I(t) x_{t-1}+\gamma_{3} \ln \sum_{0}^{t-1} x_{s}+\gamma_{4} I(t) \ln \sum_{0}^{t-1} x_{s} \\
& +\gamma_{5} x_{t-1} \ln \sum_{0}^{t-1} x_{s}+\gamma_{6} I(t) x_{t-1} \ln \sum_{0}^{t-1} x_{s}+\gamma_{7} \ln T r_{t}+w_{t}
\end{aligned}
$$

where $z_{t}=\ln x_{t}-\ln x_{t-1}, \ln T r_{t}$ is the expense of public health promotion, and $w_{t}$ is error term with zero mean and variance of $\varepsilon_{3}^{2}$ 。

\subsection{The data}

The rate of Pap smear test is collected from Pap Smear Screening Registry System Annual Report, covering the period of 1995-2009 (Bureau of Health Promotion, 2012). The rate of Pap smear test is defined as the amount of female aged 30 and above who adopt the screening. Figure 1 shows that the acceptance rate of Pap smear test grew sharply before 2001, increasing from $9.7 \%$ in 1995 to $30.2 \%$ in 2001 .

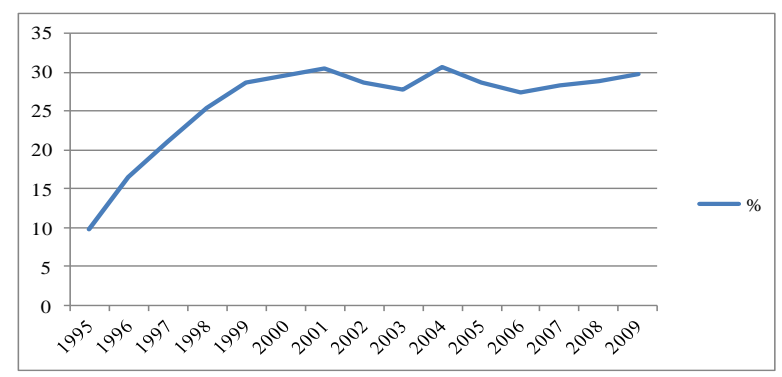

Figure 1 The rate of Pap smear test

\section{RESULTS AND DISCUSSION}

Table 1 indicates that diffusion effect affects the Pap smear test promotion significant and diffusion effect may accelerate the velocity of the innovative product spread. The organization learning effect that exists in Department of Health may enhance the performance of organization and employees will also obtain the superior ability by the accumulation of experience. The Durbin Watson test shows the specification of the models with appropriate explanation.

\subsection{Diffusion effect}

The estimation of Eq. (5) in Table 1 reveals that the amount of adopters in the earlier stage has a positive impact to the amount of adopters in the current stage. In the other words, the amount of adopters in the earlier stage increases, then the information spread will be rapider. The coefficient of variable $I_{2}$ indicates that SARS have a significant negative impact to the diffusion of Pap smear test. However, the impact of SARS is temporary and the rate of Pap smear test still maintains the increasing trend after 2004. In practice, Department of Health provides the bus to offer the medical service included cancer screening and prevention medical education to people in the rural area, and the bus also provides the House-Call service. Therefore, Pap smear test promotion is beneficiary to sustainable development and individuals can be enhanced the awareness of cervix uteri cancer prevention sustainable.

Table 1 The estimation of diffusion model, organizational

\begin{tabular}{|c|c|c|c|c|}
\hline \multirow[t]{2}{*}{ Parameters } & \multicolumn{2}{|c|}{ Diffusion model } & \multicolumn{2}{|c|}{$\begin{array}{l}\text { Organizational } \\
\text { learning model }\end{array}$} \\
\hline & Coefficient & p-value & Coefficient & p-value \\
\hline$\beta_{1}$ & $0.8162 * *$ & 0.027 & & \\
\hline$\beta_{2}$ & $0.8117 * *$ & 0.033 & & \\
\hline$\beta_{3}$ & $2.61 \mathrm{E}-06$ & 0.393 & & \\
\hline$\beta_{4}$ & $-9.1 \mathrm{E}-6^{* *}$ & 0.015 & & \\
\hline$\beta_{5}$ & $-4.5366^{*}$ & 0.094 & & \\
\hline$h_{0}$ & & & $8.9328 * *$ & $\leq 0.001$ \\
\hline$h_{1}$ & & & $0.3353 * *$ & $\leq 0.001$ \\
\hline$R^{2}$ & \multicolumn{2}{|c|}{0.7981} & \multicolumn{2}{|c|}{0.8295} \\
\hline Adjusted $R^{2}$ & \multicolumn{2}{|c|}{0.6973} & \multicolumn{2}{|c|}{0.8243} \\
\hline DW & \multicolumn{2}{|c|}{2.4968} & \multicolumn{2}{|c|}{1.5459} \\
\hline \multicolumn{5}{|c|}{ Interaction model } \\
\hline \multicolumn{3}{|l|}{ Parameters } & & \\
\hline$\gamma_{0}$ & -92.33 & 0.291 & & \\
\hline$\gamma_{1}$ & $0.0002 * *$ & 0.029 & & \\
\hline$\gamma_{2}$ & $0.0648^{* *}$ & 0.027 & & \\
\hline$\gamma_{3}$ & $5.8786^{* *}$ & $\leq 0.001$ & & \\
\hline$\gamma_{4}$ & $-1.2379 * *$ & 0.002 & & \\
\hline$\gamma_{5}$ & 0.0062 & 0.212 & & \\
\hline$\gamma_{6}$ & $-0.0033 * *$ & 0.038 & & \\
\hline$\gamma_{7}$ & 1.5455 & 0.873 & & \\
\hline$R^{2}$ & 0.7895 & & & \\
\hline Adjusted $R^{2}$ & 0.7791 & & & \\
\hline DW & 2.4297 & & & \\
\hline
\end{tabular}
learning model, and interaction model

\subsection{Organizational learning effect}

Eq. (7) can compute the organizational learning rate by using $\mathrm{LR}=\exp \left(h_{1}\right)$ as 1.3983 . Table 1 shows that organizational learning may be generated and applied by the interaction of employee. Therefore, the institution factors included the process of information exchange and experience accumulation that will effectively improve the performance of employee may imply the high learning rate. MartínRojas et al. (2011) indicate that organizational learning may maintain a system of knowledge creation and enhance the performance of organization. In practice, Department of Health has taken into the consideration of investment on learning system including employee education, cancer screening promotion program, on-job 
training, cancer screening knowledge promotion programs, etc.

\subsection{Interaction effect}

The estimation of Eq. (9) in Table 1 reveals that the diffusion of Pap smear test interacts with the organizational learning and it also indicates that the interaction between diffusion effect and organizational learning effect is a determinant factor to affect the promotion of Pap smear test. Due to the increment of the opportunity for assimilating the information and education about Pap smear test, the amount of female who decide to accept the service of Pap smear test will be affected. Barreto and Kemp (2008) propose that requirements and opportunities from the early adopters is an important factor to affect the process of diffusion and the rate of innovation adoption decided by the number of adopters. Through the support of the empirical results and observation in this paper, we conclude that the promotion of Pap smear test may attribute to the diffusion effect, the organizational effect and the interaction effect.

In order to achieve the goal of high rate of Pap smear test, Department of Health devote the integration of medical resource included the connection of medical network that collect all the patient information and can be transfer between Division of Disease Control and Prevention, Health Promotion Division, distinct health center, hospitals, and public health service center. In the other word, the integration of medical resource can be treated as a system that will transfer the information of patients between each other. If the female who aged 30 or above and has not received Pap smear test in this system, the information will transfer between the medical network and inform you to accept Pap smear test. The creation of "system" arrangement with females in fact works effectively and enhances the opportunity of the assimilation of cancer prevention for female.

In this paper, we propose three models to describe the promotion of Pap smear test. Information about the innovative products (Pap smear test) will be exchanged in the social network and accepted by the public in a society. In practice, females (the users and the potential adopters) may assimilate the information about the Pap smear test by personal contacts. On the other hand, organizational learning effect occurs in Department of Health is encouraged to be designed a better mechanism for provision of the information about Pap smear test in the social network. The results by the estimation of interaction effect model show that the positive relationship between organizational learning effect and diffusion effect, and imply the performance of Department of Health may affect the females' adoption of Pap smear test service provided by this system.

\section{CONCLUSION}

In this paper, we integrate the diffusion effect and the organizational learning effect to describe the integrative relationship between the Department of Health and females. The results also show that the diffusion effect, organizational learning effect and interaction effect play an important role to affect the promotion of Pap smear test. In practice, it is an appropriate way to promote the Pap smear test by integrating the diffusion effect associated with the females' support and organizational learning determined by Department of Health. According to the results, it is important to improve the interlinking of Department of Health and female for participating the cancer prevention. Therefore, Department of Health needs to design a strategy for integrating the medical resource that may improve the process of information dissemination.

\section{REFERENCES}

[1] Barreto, L., Kemp, R. (2008). Inclusion of technology diffusion in energy-systems models: some gaps and needs, Journal of Cleaner Production, 16(S1), S95-S101.

[2] Bayus, B. L. (1993). High-definition television: assessing demand forecasts for a next generation consumer durable, Management Science, 39 (11), 1569-1585.

[3] Bolívar-Ramos, M. T., García-Morales, V.J., GarcíaSánchez, E. (2012). Technological distinctive competencies and organizational learning: Effects on organizational innovation to improve firm performance. J. Eng. Technol. Manage, 29, 331-357.

[4] Bureau of Health Promotion (2012). Pap smear screening registry system annual report. Department of Health, Executive Yuan. http://www.bhp.doh.gov.tw

[5] Chen, L. S., Huang, N., Tsay, J. H., Wang, P. J., Chou, Y. J., Chou, P., Lee, C. H. (2007). Screening for cervical cancer among female physicians and their relatives in Taiwan - A population-based comparative study, Preventive Medicine, 44, 531-535.

[6] Chen, Y. T. Chang, D. S. (2010). Diffusion effect and learning effect: an examination on MSW recycling, Journal of Cleaner Production, 18, 496-503.

[7] Kemp, R. (1998). The diffusion of biological waste-water treatment plants in the Dutch food and Beverage industry, Environmental and Resource Economics, 12, 113-136.

[8] Meyer, P. S., Ausbel, J. H. (1999). Carrying capacity: a model with logistically varying limits, Technological Forecasting and Social Change, 61 (3), 209-214.

[9] Martín-Rojas, R., García-Morales, V. J., García-Sánchez, E. (2011). The influence on corporate entrepreneurship of technological variables, Industrial Management \& Data Systems, 111 (7), 984-1005.

[10] Montalvo, C., Kemp, R., (2008). Cleaner technology diffusion: Case studies, modeling and policy, Journal of Cleaner Production, 16 (S1), S1-S6.

[11] Rogers, E. M. (2003). Diffusion of Innovation, 4th ed., The Free Press, New York.Sohn, S. Y., Ahn, B. J. (2003). Multigeneration diffusion model for economic assessment of new technology, Technological Forecasting \& Social Change, 70, 251-264. 